\title{
Jastrow-correlated wave functions for flat-band lattices
}

\author{
Hao Wang and V. W. Scarola \\ Department of Physics, Virginia Tech, Blacksburg, Virginia 24061, USA
}

(Received 28 April 2011; published 20 June 2011)

\begin{abstract}
The electronic band structure of many compounds, e.g., carbon-based structures, can exhibit essentially no dispersion. Models of electrons in flat-band lattices define nonperturbative strongly correlated problems by default. We construct a set of Jastrow-correlated ansatz wavefunctions to capture the low energy physics of interacting particles in flat bands. We test the ansatz in a simple Coulomb model of spinless electrons in a honeycomb ribbon. We find that the wavefunction accurately captures the ground state in a transition from a crystal to a uniform quantum liquid.
\end{abstract}

DOI: 10.1103/PhysRevB.83.245109

PACS number(s): 73.22.-f, 71.10.Pm

\section{INTRODUCTION}

Quantum correlated phases and rich phase diagrams can manifest even in systems with very little contribution from the kinetic energy. The long-range part of the interaction itself can lead to interesting and unexpected physics. Hubbard's work on lattice models found that the long-range part of the Coulomb interaction alone supports insulating crystals that stabilize at odd denominator lattice fillings. ${ }^{1}$ His analysis brings up an interesting question: Can interactions by themselves lead to new and interesting physics?

Particles hopping with an otherwise large kinetic energy can interfere to show no dispersion in certain lattices geometries (see, e.g., Ref. 2). Such flat bands arise as particles hop among a few sites and interfere to form localized states. At first, one would expect such lattices to exhibit only classical localization effects. However, the absence of any dispersion leaves the interaction as the de facto dominant energy scale thus allowing interesting quantum many-body effects to appear solely from off-diagonal contributions in the interaction or, equivalently, from noncommuting lattice density operators.

The paucity of theoretical work on interacting flat-band systems stems from implicit difficulties in solving even the simplest models. Vanishing band curvature implies very little or no screening of the interaction. Off-diagonal terms in the interaction can therefore be relevant in such systems. Furthermore, the absence of dispersion results in a single dominant term in the simplest flat-band models: $H_{\mathrm{FB}}=$ $\mathcal{P}_{\mathrm{FB}}^{\dagger} V \mathcal{P}_{\mathrm{FB}}$, where $\mathcal{P}_{\mathrm{FB}}$ projects into a flat band and $V$ is a two-body interaction. With no small parameter, conventional perturbative methods fail. Work on flat-band lattices has become more pressing with the discovery of systems hosting flat bands.

The discovery of carbon based nanostructures, e.g., carbon nanotubes ${ }^{3}$ and graphene, ${ }^{4}$ opened the possibility of a geometric tuning of electronic band structure. Detailed calculations show flat bands in a variety of physically relevant contexts, including: the edges of two-dimensional (2D) graphene, ${ }^{5}$ one-dimensional (1D) graphene nanoribbons, ${ }^{5-7}$ hydrogenated graphene nanoribbons, ${ }^{8}$ collapsed carbon nanotubes, ${ }^{9}$ hydrogenated nanotubes, ${ }^{10}$ graphene dots, ${ }^{11}$ and graphene antidots. ${ }^{12}$ Flat bands can also be found in a wealth of other compounds and engineered in optical lattices. ${ }^{13}$ The ubiquity of flat-band systems imply that strong interaction effects demand further study.
We propose that studies in an entirely different context, the quantum Hall regime, can shed light on the problem of strong correlation in flat-band lattices. Models of the quantum Hall effects project into a single Landau band in the high field limit. The degenerate Landau level leaves the Coulomb interaction as the sole term in the simplest models to yield direct analogies with models of flat-band lattices in the absence of a magnetic field.

Parallels between quantum Hall and zero-field lattice formalisms have been drawn at the Hamiltonian level. Early work $^{14,15}$ compared models of Laughlin's states ${ }^{16}$ in the cylindrical geometry ${ }^{17}$ to lattice models of spinless fermions. Here, it was shown that, on thin cylinders, components of Laughlin's wavefunction can be thought of in terms of Hubbard's classical crystals. ${ }^{1}$ The work on thin cylinders marked a key advance by noting an implicit 1D structure in quantum Hall models. Subsequent analyses explored intriguing aspects of this lattice-quantum Hall connection. ${ }^{18}$

We argue that a connection between flat-band and quantum Hall formalisms can be drawn at the level of ansatz wave functions. Elegant but accurate many-body wavefunctions quantitatively model the physics of the quantum Hall regime. ${ }^{16,19,20}$ Here, we build a similar class of Jastrowcorrelated wave functions ${ }^{21}$ as ansatz states for flat-band lattices. We numerically test a quasi-1D example in a toy model of a flat band in graphene nanoribbons (Fig. 1) of up to 476 sites. We find that the ansatz state accurately captures the ground state in a remarkable transition from a Wigner crystal to a uniform quantum liquid driven entirely by interactions.

\section{JASTROW-CORRELATED ANSATZ WAVEFUNCTIONS}

We construct Jastrow-correlated first quantized wavefunctions, applicable to flat-band lattices. We consider a family of states motivated by Jastrow-correlated states written in the lowest Landau level. ${ }^{16,19}$ For a generic two-body interaction that depends only on relative separation, $H_{\mathrm{FB}}$ commutes with the center of mass operator. We may therefore construct states of the form, $\Psi_{\mathrm{CM}} \Psi_{\text {rel }}$, where $\Psi_{\mathrm{CM}}$ and $\Psi_{\text {rel }}$ are functions of the center of mass and relative coordinates, respectively. We propose that the following set of unnormalized relative-coordinate wavefunctions are energetically favorable ansatz states for a broad class of flat-band lattice 


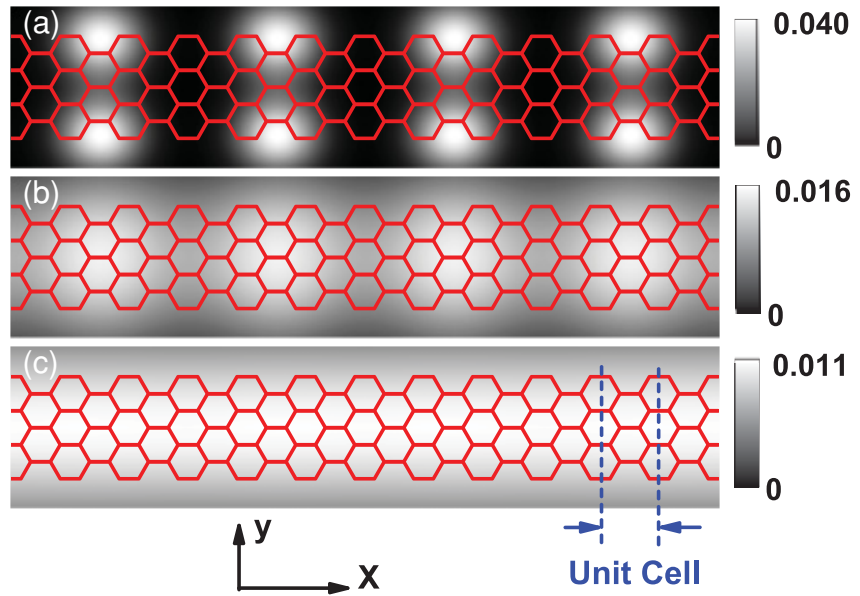

FIG. 1. (Color online) Grayscale plot of numerically calculated solutions for the electron density of the flat-band Coulomb problem in a honeycomb ribbon with $N=12$ electrons and $N_{x}=34$ unit cells zoomed into 12 central unit cells. Sites sit at vertices in the depiction of the underlying lattice. The three graphs show three distinct states that arise from increasing width parameter of basis states: (a) Wigner crystal ( $\beta=2.0)$, (b) charge density wave $(\beta=3.5)$, and (c) uniform state $(\beta=5.0)$.

models:

$$
\left|\Psi_{\text {rel }}^{\nu}\right\rangle=\prod_{j<k,\{\Lambda\}}\left(a_{j, \Lambda}^{\dagger}-a_{k, \Lambda}^{\dagger}\right)^{m_{c}}\left|\psi^{\nu^{*}}\right\rangle,
$$

where the symbol $\{\Lambda\}$ denotes a set of 1D chains that cover a bravais lattice. Each bond-oriented chain uniquely covers bonds and the two end sites lie at the lattice edges. The first quantized operator for particle $j,\left(a_{j, \Lambda}^{\dagger}\right)^{n}$ creates a state localized at the position $\mathbf{r}_{n}^{\Lambda}=\delta_{\Lambda}+n \mathbf{b}_{\Lambda}$, where $n \in \mathbb{N}$ and $\delta_{\Lambda}$ is the position vector for a unique edge starting site of the chain $\Lambda$ (e.g., $\delta=0$ for a 1D lattice and $\{\delta\}=\left\{\left(n_{x}^{0}, n_{y}^{0}\right),\left(n_{x}^{0} \cdot n_{y}^{0}=\right.\right.$ $0)\}$ for a square lattice). The set of bond vectors $\mathbf{b}_{\Lambda}$ point from the starting site to a nearest neighbor site along the chain. (This chain covering procedure must be modified for some nonbravais lattices.) We define real space single particle basis states as $\left\langle\mathbf{r}\left|\left(a_{\Lambda}^{\dagger}\right)^{n}\right| 0\right\rangle=\sqrt{n !} f_{n}(\gamma) w\left(\mathbf{r}-\mathbf{r}_{n}^{\Lambda}\right)$, where $w$ are Wannier functions. $f$ is a variational function. For the model studied here we choose $f_{n}(\gamma)=\exp \left(\gamma^{2} n^{2} / 2\right) / \sqrt{n !}$, where $\gamma$ is a variational parameter.

The Jastrow factor in Eq. (1) enforces Gutzwiller projection. It attaches an integer number of correlation holes, $m_{c}$, to each particle in the wave function $\psi^{v^{*}}$ at a filling, $v^{*}$, to thereby form a state at the reduced filling $v=v^{*} /\left(m_{c} v^{*}+1\right)$, akin to a procedure introduced in the quantum Hall regime. ${ }^{19}$ Here, $v$ refers to the number of particles per basis state. In the following we consider fermions. The choice $m_{c}=2 p$ with antisymmetric $\psi^{\nu^{*}}$ preserves the antisymmetry of $\Psi_{\text {rel }}$.

In the following, we test a 1D example of Eq. (1) and a specific choice for $\psi^{\nu^{*}}$. We consider a Hartree-Fock state, $\psi^{v^{*}=1}=\operatorname{Det}\left[\left(a_{i}^{\dagger}\right)^{n}\right]|0\rangle=\prod_{j<k}\left(a_{j}^{\dagger}-a_{k}^{\dagger}\right)|0\rangle$. With this choice, the relative coordinate wavefunction takes a form similar to the Laughlin state: ${ }^{16,22}$

$$
\left|\Psi_{1 \mathrm{D}-\text { rel }}^{\nu}\right\rangle=\prod_{j<k}\left(a_{j}^{\dagger}-a_{k}^{\dagger}\right)^{2 p+1}|0\rangle .
$$

For an $N$-particle system, the number of particles per basis state is then $v=N /[(2 p+1) N-2 p]$. We focus on $p=1$, which corresponds to $v=1 / 3$ for $N \rightarrow \infty$.

The variational parameter, $\gamma$, tunes the above wavefunctions between distinct limits. For $\gamma \rightarrow \infty$, the wavefunctions describe crystals (Wigner crystals) because the basis states are highly localized. For intermediate values, $\gamma \sim 1$, we have a charge density wave. For $\gamma \rightarrow 0$, we obtain uniform liquid states. To see this one notes that the Wannier functions can be written in terms of polynomials in Fourier transform space. ${ }^{23}$ For a $1 \mathrm{D}$ lattice along $x$, we have $w\left(x-x_{n}\right)=$ $\mathcal{F} \exp (i q n) \tilde{w}(q)$, where $\mathcal{F}$ denotes the Fourier integral over all $q$-space and $\tilde{w}(q)$ is the Fourier transform of the Wannier function at the edge. Then, Eq. (2) becomes a Laughlin-like state:

$$
\lim _{\gamma \rightarrow 0} \Psi_{1 \mathrm{D} \text {-rel }}^{\nu}=\mathcal{F} \prod_{j<k}\left(e^{i q_{j}}-e^{i q_{k}}\right)^{2 p+1} \prod_{j} \tilde{w}\left(q_{j}\right),
$$

which describes a uniform quantum liquid.

\section{MODEL OF INTERACTING FERMIONS IN A FLAT BAND}

We test the validity of Eq. (2) as an ansatz ground state for a model of $N$ spinless fermions interacting through a truncated Coulomb interaction in a flat band of a honeycomb ribbon. We consider a second quantized flat-band model:

$$
H=\frac{1}{2} \sum_{n_{1} n_{2} n_{3} n_{4}} V_{\{n\}} \hat{c}_{n_{1}}^{\dagger} \hat{c}_{n_{2}}^{\dagger} \hat{c}_{n_{3}} \hat{c}_{n_{4}},
$$

where the matrix elements $V_{\{n\}}$ are determined by the form of the interaction $V\left(\left|\mathbf{r}-\mathbf{r}^{\prime}\right|\right)$ and flat-band basis states $\phi$ as

$$
V_{\{n\}}=\int d \mathbf{r} d \mathbf{r}^{\prime} \phi_{n_{1}}^{*}(\mathbf{r}) \phi_{n_{2}}^{*}\left(\mathbf{r}^{\prime}\right) V\left(\left|\mathbf{r}-\mathbf{r}^{\prime}\right|\right) \phi_{n_{3}}\left(\mathbf{r}^{\prime}\right) \phi_{n_{4}}(\mathbf{r}) .
$$

The second quantized operator $\hat{c}_{n}^{\dagger}$ creates a fermion in the state $\phi_{n}$.

Flat-band basis states follow from the lattice structure. In the following, we work in units of the honeycomb bond length, $a_{0}=1$, and Coulomb energy, $e^{2} / \epsilon a_{0}\left(\sim 10^{5} \mathrm{~K}\right.$ for a carbon bond in vacuum). We consider a quasi-1D honeycomb ribbon of width $L_{y}=\sqrt{3} N_{y}$ along the $y$ direction. Along the ribbon, we allow the range of $x$ to be infinite, but restrict the centers of local electron orbitals to lie in $N_{x}$ unit cells along a length of $L_{x}=3 N_{x}$. For simplicity, we assume Gaussians of width $\sim \beta$ localized at each site of the honeycomb lattice. The hopping energy between sites can be used to determine $\beta$ (or vice versa) prior to projection into the flat band. In the honeycomb geometry, the flat-band basis states are

$$
\phi_{n}(\mathbf{r})=N_{\beta} \sum_{s=1}^{2 N_{y}} t_{s} e^{-\left(\mathbf{r}-\mathbf{R}_{n}^{s}\right)^{2} / 2 \beta^{2}}, \quad\left(n=1, \ldots, N_{x}\right),
$$

where $N_{\beta}$ is a normalization constant, $\mathbf{R}_{n}^{s}=\left(X_{n}^{s}, Y^{s}\right)$ is the location of the $s$ th node in the $n$th unit cell, and the node factor $t_{s}$ has the form of $t_{2 u-1}=t_{2 u}=(-1)^{u+1}$ for $u=$ $1, \ldots, N_{y} .{ }^{6}$ The node locations arising from the underlying honeycomb structure are $\mathbf{R}_{n}^{s}=\left(3 n-\epsilon_{s}, \sqrt{3}(u-1 / 2)\right)$, where $\epsilon_{s}=2\left(\epsilon_{s}=1\right)$ for $s=2 u-1(s=2 u)$ and $u=1, \ldots, N_{y}$. In the following, we set $N_{y}=3$. Using the above Wannier state 
$\phi_{n}$ as a single-particle basis state localized at the $n$th unit cell, we can construct the interaction matrix elements analytically.

We stress that the model considered here idealizes flat bands of armchair nanoribbons. The Gaussian localized states chosen in Eq. (6) are only convenient approximations for orbitals of electrons in flat bands of suspended graphene nanoribbons. For example, the $\pi_{z}$ Slater orbitals of carbon atoms could be used instead. ${ }^{7}$ These orbitals are, in contrast, exponentially localized and loosely correspond to $\beta \sim 1$. We expect less localization (larger $\beta$ ) in carbon structures with adsorbates (e.g., hydrogen ${ }^{8}$ ). It is straightforward to replace the Gaussian orbitals in our formalism with density functional theory results for specific systems to make concrete predictions for experiments. Furthermore, we have ignored effects from nearby bands.

We now compute the interaction matrix elements in the flat-band basis with Gaussian orbitals. The Fourier expansion of the interaction is $V(r)=e^{i y Q_{0}} \int d Q V(Q) e^{i Q x} / \pi$ with $Q_{0}=\pi / \sqrt{3}$ and $V(Q)=\left(e^{2} / \epsilon\right) \sum_{m} V_{m} L_{m}\left(Q^{2}+Q_{0}^{2}\right)$, were the $L_{m}$ are the Laguerre polynomials, $m$ is an odd integer for spinless fermions, and $V_{m}=\sqrt{\pi}(2 m-1) ! ! /\left(2^{m+1} m !\right)$ is a pseudopotential parameter for the full Coulomb interaction. ${ }^{24}$ We choose only the $m=1$ term, which corresponds to an effective short-range interaction $V(r) \sim \nabla^{2} \delta(\mathbf{r})$. The matrix elements are then

$$
V_{\{n\}}=\sum_{\{s\}} T_{\{s\}} e^{-\left(r_{n_{1} n_{4}}^{2}+r_{n_{2} n_{3}}^{2}+\Delta R^{2} / 2\right)}\left(\frac{\Delta R^{2}}{\beta^{3}}-\frac{\alpha}{\beta}\right),
$$

where we define

$$
\begin{gathered}
T_{\{s\}}=V_{1} T_{s_{1} s_{4}} T_{s_{2} s_{3}}^{*} /\left(\sqrt{2 \pi} N_{\beta}^{4}\right), \\
T_{s s^{\prime}}=t_{s} t_{s^{\prime}} \int_{0}^{L_{y}} d y e^{\left[i Q_{0} y-\left(\left(y-Y^{s}\right)^{2}+\left(y-Y^{s^{\prime}}\right)^{2}\right) /\left(2 \beta^{2}\right)\right]}, \\
r_{n_{1} n_{4}}=\left(X_{n_{1}}^{s_{1}}-X_{n_{4}}^{s_{4}}\right) /(2 \beta), \\
r_{n_{2} n_{3}}=\left(X_{n_{2}}^{s_{2}}-X_{n_{3}}^{s_{3}}\right) /(2 \beta), \\
\Delta R=\left(X_{n_{1}}^{s_{1}}+X_{n_{4}}^{s_{4}}-X_{n_{2}}^{s_{2}}-X_{n_{3}}^{s_{3}}\right) /(2 \beta), \\
\alpha=\beta^{-2}+Q_{0}^{2} .
\end{gathered}
$$

\section{NUMERICAL TEST OF ANSATZ}

We test our ansatz by solving Eqs. (4)-(8) with numerical diagonalization on finite-sized ribbons. We restrict our ground state to the Hilbert space with the center of mass at the center of the ribbon. In the thermodynamic limit, the center of mass is a good quantum number but in finite sized systems we omit edge effects in extreme unit cells by restricting our basis. We test several different system sizes and show below that our results have converged with increasing system size.

Figure 1 plots a zoom in of the quasi-1D charge density over a few central unit cells of an $N=12$ and $N_{x}=34$ system computed from our flat-band model, Eqs. (4)-(8). The charge density is $\rho(\mathbf{r})=\sum_{n=1}^{N_{x}}\left|\phi_{n}(\mathbf{r})\right|^{2} \rho_{n}$, where $\rho_{n}=$ $\left\langle\Psi_{\mathrm{FB}}\left|\hat{c}_{n}^{\dagger} \hat{c}_{n}\right| \Psi_{\mathrm{FB}}\right\rangle$ is the local density of the $n$th unit cell and $\Psi_{\mathrm{FB}}$ is the exact ground state. The density is plotted against the underlying honeycomb lattice to show that each unit cell consists of 14 sites in this example. Figure 1 shows the evolution from a Wigner crystal configuration (a), to a charge

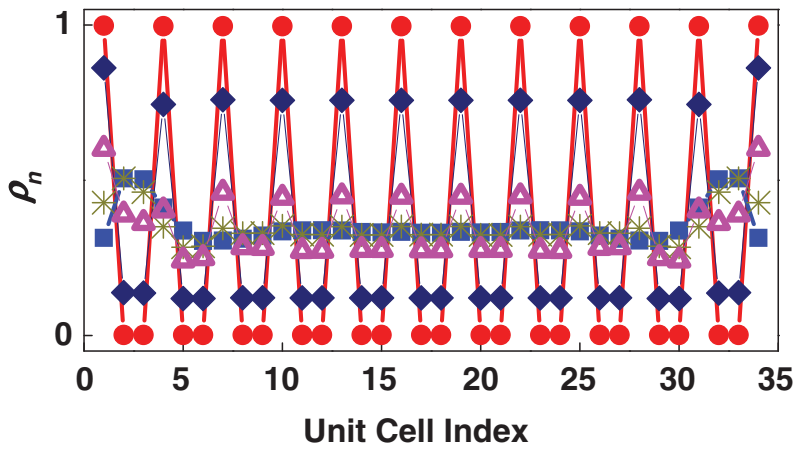

FIG. 2. (Color online) The local density of each unit cell, $\rho_{n}$, plotted versus the unit cell index along the honeycomb ribbon with $N=12$ particles. The circles, diamonds, triangles, stars, and squares are obtained from width parameters $\beta=2,3,4,5$, and 6 , respectively. The lines are a guide to the eye.

density wave (b), and then to a uniform state (c) as we increase the extent of basis states. The same features are exhibited in Fig. 2, the local density plot for each unit cell.

We check the validity of Eq. (2) in describing the ground state of Eqs. (4)-(8) by taking overlaps. We generate Eq. (2) numerically by noting that it is the exact eigenstate of a model of the lowest Landau level of electrons on a cylinder. ${ }^{15,17} \mathrm{We}$ obtain the maximum overlap with the choice $\gamma=3 / \beta$. The right axis of Fig. 3 plots the overlap of Eq. (2) with the ground state of Eqs. (4)-(8). The left axis plots the deviation in the local density from the center to the nearest neighboring unit cell, $\Delta \rho_{\mathrm{c}} \equiv\left|\rho_{\left(N_{x} / 2\right)}-\rho_{\left(N_{x} / 2-1\right)}\right| . \Delta \rho_{\mathrm{c}}=1$ and 0 correspond for a crystal and a uniform state, respectively. We find that the overlap is essentially constant and larger than $99.61 \%$ for all $\beta$ thus showing that the ansatz wavefunction accurately captures the ground state of the truncated Coulomb interaction in all parameter regimes. We expect the overlap to decrease as we increase the range of the interaction or change the form of the Wannier function. Overlaps can be improved by choosing a better variational form for $f(\gamma)$.

It is interesting to note that the ansatz state adiabatically connects the Wigner crystal to a uniform, Jastrowcorrelated liquid state based on the Laughlin wavefunction. This crossover was pointed out in the quantum Hall context

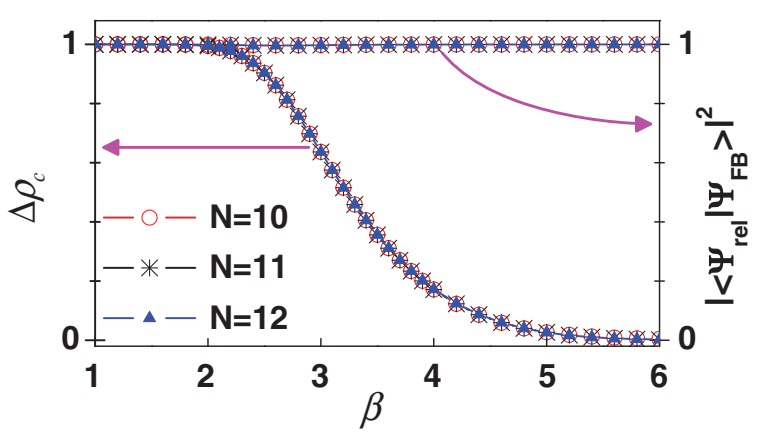

FIG. 3. (Color online) The left axis plots the deviation in local density between neighboring unit cells at the sample center versus the basis state width parameter for three different system sizes. The right axis plots the overlap between the ansatz state, Eq. (2), and the numerically computed ground state of Eqs. (4)-(8). 


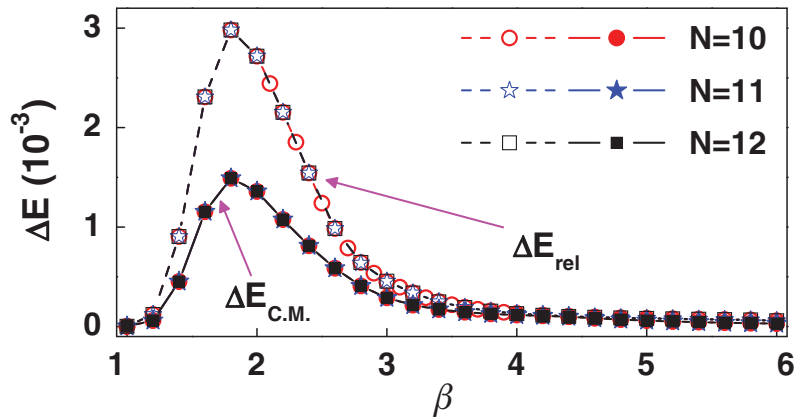

FIG. 4. (Color online) The excitation energy plotted versus the basis state width parameter for three different system sizes. Open (closed) symbols indicate the energy gap in the relative (center of mass) degrees of freedom.

in Ref. 15. Here, we have shown that a mapping of the quantum Hall state to a zero-field flat-band lattice preserves this crossover.

Figure 4 plots the energy gap as a function of $\beta$. In an infinitely large system, excitations can either leave the center of mass intact (excitations in the relative coordinate degrees of freedom) or shift the center of mass (center of mass excitations). We find a small but finite gap for the uniform state (large $\beta$ ) in the relative degrees of freedom. The gap appears to remain robust against system size suggesting that the gap we have computed is indeed a bulk gap, thus signaling the intriguing possibility of an incompressible uniform state. We note, however, that the center of mass gap becomes comparable to the relative coordinate gap for large $\beta$. It is possible that excitations in the relative degrees of freedom are spatially large in this case and therefore sensitive to edge effects. Periodic systems will allow a more accurate estimate of the bulk gap.

\section{SUMMARY}

We have constructed a general class of Jastrow-correlated wavefunctions applicable to flat-band lattices. We studied a toy model of spinless fermions in a flat band in graphene nanoribbons in the absence of a magnetic field. We find that the wavefunction accurately captures an intriguing transition from a Wigner crystal to a Jastrow correlated liquid, driven entirely by interactions. Our method can be generalized to accurately model graphene nanoribbons and other flatband lattice structures ${ }^{5-13}$ and can be adapted to study magnetism recently explored in experiments on graphene nanoribbons. $^{25}$

The Jastrow-correlated wavefunctions constructed here are versatile. They can be combined with density functional theory estimates of Wannier functions to simultaneously capture band structure and the effects of strong interactions. They can be generalized to apply to a wide variety of lattices, describe correlated bosons, or incorporate spin. For example, $\Psi_{\text {rel }}$ can quantify a connection between GutzwillerRVB and quantum Hall states. ${ }^{26}$ Gutzwiller projected $d$ wave spin-singlet paired states in a $2 \mathrm{D}$ square lattice can be constructed by writing $\psi^{v^{*}}$ as an antisymmetric product of singlet paired states, $\propto \mathcal{A}\left[g\left(\mathbf{r}_{1}-\mathbf{r}_{2}\right) g\left(\mathbf{r}_{3}-\mathbf{r}_{4}\right) \ldots\right]$. Here, an effective pairing among $\psi^{\nu *}$ particles could arise from an over-screening of long range repulsion, as in the quantum Hall regime, ${ }^{20,27,28}$ but in flat or narrow-band lattice models.

\section{ACKNOWLEDGMENTS}

We thank K. Park for helpful comments and the Thomas F. Jeffress and Kate Miller Jeffress Memorial Trust, Grant No. J-992, for support.
${ }^{1}$ J. Hubbard, Phys. Rev. B 17, 494 (1978).

${ }^{2}$ H. Tasaki, Prog. Theor. Phys. 99, 489 (1998).

${ }^{3}$ S. Iijima, Nature (London) 354, 56 (1991).

${ }^{4}$ K. S. Novoselov, A. K. Geim, S. V. Morozov, D. Jiang, Y. Zhang, S. V. Dubonos, I. V. Grigorieva, and A. A. Firsov, Science 306, 666 (2004).

${ }^{5}$ K. Nakada, M. Fujita, G. Dresselhaus, and M. S. Dresselhaus, Phys. Rev. B 54, 17954 (1996).

${ }^{6}$ H.-H. Lin, T. Hikihara, H.-T. Jeng, B.-L. Huang, C.-Y. Mou, and X. Hu, Phys. Rev. B 79, 035405 (2009).

${ }^{7}$ P. Potasz, A. D. Güçlü, and P. Hawrylak, Phys. Rev. B 82, 075425 (2010).

${ }^{8}$ K. Kusakabe and M. Maruyama, Phys. Rev. B 67, 092406 (2003).

${ }^{9}$ P. E. Lammert, P. Zhang, and V. H. Crespi, Phys. Rev. Lett. 84, 2453 (2000)

${ }^{10}$ X. Pei, X. Yang, and J. Dong, Phys. Rev. B 73, 195417 (2006); X. Yang and G. Wu, ACS Nano 3, 1646 (2009).

${ }^{11}$ M. Ezawa, Phys. Rev. B 76, 245415 (2007); J. Fernández-Rossier and J. J. Palacios, Phys. Rev. Lett. 99, 177204 (2007); A. D. Güçlü, P. Potasz, O. Voznyy, M. Korkusinski, and P. Hawrylak, ibid. 103, 246805 (2009).
${ }^{12}$ M. Vanević, V. M. Stojanović, and M. Kindermann, Phys. Rev. B 80, 045410 (2009); J. A. Fürst, T. G. Pedersen, M. Brandbyge, and A. P. Jauho, ibid. 80, 115117 (2009).

${ }^{13}$ C. J. Wu, D. Bergman, L. Balents, and S. Das Sarma, Phys. Rev. Lett. 99, 070401 (2007).

${ }^{14}$ E. Westerberg and T. H. Hansson, Phys. Rev. B 47, 16554 (1993).

${ }^{15}$ E. H. Rezayi and F. D. M. Haldane, Phys. Rev. B 50, 17199 (1994).

${ }^{16}$ R. B. Laughlin, Phys. Rev. Lett. 50, 1395 (1983).

${ }^{17}$ D. J. Thouless, Surf. Sci. 142, 147 (1984).

${ }^{18}$ D. H. Lee and J. M. Leinaas, Phys. Rev. Lett. 92, 096401 (2004); E. J. Bergholtz and A. Karlhede, ibid. 94, 026802 (2005); A. Seidel, H. Fu, D.-H. Lee, J. M. Leinaas, and J. Moore, ibid. 95, 266405 (2005); S. Jansen, E. H. Lieb, and R. Seiler, Phys. Status Solidi B 245, 439 (2008).

${ }^{19}$ J. K. Jain, Phys. Rev. Lett. 63, 199 (1989).

${ }^{20}$ Perspectives in Quantum Hall Effects, edited by S. Das Sarma and A. Pinczuk (Wiley, New York, 1997); J. K. Jain, Composite Fermions (Cambridge University Press, Cambridge, 2007).

${ }^{21}$ R. Jastrow, Phys. Rev. 98, 1479 (1955).

${ }^{22}$ S. M. Girvin and T. Jach, Phys. Rev. B 28, 4506 (1983).

${ }^{23}$ J. C. Slater, Phys. Rev. 87, 807 (1952). 
${ }^{24}$ F. D. M. Haldane, Phys. Rev. Lett. 55, 2095 (1985).

${ }^{25}$ C. Tao, L. Jiao, O. V. Yazyev, Y. Chen, J. Feng, X. Zhang, R. B. Capaz, J. M. Tour, A. Zettl, S. G. Louie, H. Dai, and M. F. Crommie, Nature Phys. advanced online publication, 08 May 2011, doi:10.1038/nphys1991.
${ }^{26}$ J. K. Jain and P. W. Anderson, Proc. Natl. Acad. Sci. USA 106, 9131 (2009).

${ }^{27}$ N. Read and D. Green, Phys. Rev. B 61, 10267 (2000).

${ }^{28}$ V. W. Scarola, K. Park, and J. K. Jain, Nature (London) 406, 863 (2000). 